University of Nebraska - Lincoln

DigitalCommons@University of Nebraska - Lincoln

January 2007

\title{
Electromechanical Behavior in Biological Systems at the Nanoscale
}

\author{
Alexei Gruverman \\ University of Nebraska-Lincoln, agruverman2@unl.edu \\ Brain J. Rodriguez \\ Oak Ridge National Laboratory, brian.rodriguez@ucd.ie \\ Sergei Kalinin \\ Oak Ridge National Laboratory, sergei2@ornl.gov
}

Follow this and additional works at: https://digitalcommons.unl.edu/physicsgruverman

Part of the Physics Commons

Gruverman, Alexei; Rodriguez, Brain J.; and Kalinin, Sergei, "Electromechanical Behavior in Biological Systems at the Nanoscale" (2007). Alexei Gruverman Publications. 42.

https://digitalcommons.unl.edu/physicsgruverman/42

This Article is brought to you for free and open access by the Research Papers in Physics and Astronomy at DigitalCommons@University of Nebraska - Lincoln. It has been accepted for inclusion in Alexei Gruverman Publications by an authorized administrator of DigitalCommons@University of Nebraska - Lincoln. 
Published in:

Scanning Probe Microscopy: Electrical and Electromechanical Phenomena at the Nanoscale, Sergei Kalinin and Alexei Gruverman, editors, 2 volumes (New York: Springer Science+Business Media, 2007).

$\begin{array}{lllllll}\text { D } & \mathbf{0} & \mathbf{0} & \mathbf{0} & \mathbf{0} & \mathbf{0} & \mathbf{0}\end{array}$

Alexei Gruverman, University of Nebraska-Lincoln

Brian J. Rodriguez, Oak Ridge National Laboratory

Sergei Kalinin, Oak Ridge National Laboratory

This document is not subject to copyright. 


\title{
III.3
}

\section{Electromechanical Behavior in Biological Systems at the Nanoscale}

\author{
A. GRUVERMAN, B. J. Rodriguez, AND S. V. KALININ
}

Hierarchical structure of connective and calcified tissues from the macro- to nanoscale level determines the mechanical and biological functionality of biological materials and has been the focus of numerous recent studies. Further progress in this field requires development of microscopic techniques capable of probing materials properties, including local composition, crystallographic orientation, and mechanical properties on the nanometer-length scale. Here, we describe a piezoresponse force microscopy (PFM) approach to high-resolution imaging of biological systems, based on detection of the local piezoelectric response. Samples include human tooth, femoral cartilage, deer antler, and butterfly wing scales. PFM allows differentiation between organic and mineral components and provides additional important information on materials microstructure. We also demonstrate the PFM capability of studying the internal structure and orientation of protein microfibrils with a spatial resolution of several nanometers. Future potential of the PFM approach for biological imaging is discussed.

\section{Introduction}

Since the discovery in the late 18 th century of electrically induced mechanical response in muscle tissue, coupling between electrical and mechanical phenomena has been shown to be a near-universal feature of biological systems. This observation provided generations of scientists with tangible justification for studying the effect of electrical stimulus on the mechanical functioning of biological tissues that eventually led to the beginning of such scientific disciplines as electrophysiology and bioelectrochemistry. One of the most important manifestations of electromechanical coupling in biological systems is piezoelectricity, which stems from the crystal structure of most biopolymers including cellulose, collagen, keratin, chitin, amylose, and DNA. Piezoelectricity has been found in a number of biological systems including bones, teeth, wood, proteins, polysaccharides, etc. [1-3]. After the seminal work of Fukada [4-6] the main motivation of studying piezoelectricity in biosystems became understanding the relationship between physiologically 
generated electric fields and mechanical properties on the molecular, cellular, and tissue levels.

Early investigations of the piezoelectric properties of biological systems have been performed on the macroscopic level, similarly to conventional mechanical and dielectric measurements. These studies were mainly based on the detection of the direct piezoelectric effect in macroscopic samples (typically chosen to conform to simple cubic or rectangular shape) as a function of temperature, humidity, frequency, and sample orientation. It has been noticed that measurements of the electrical signal in the physiological sample are prone to multiple artifacts related to finite conductivity and the presence of mobile ions, when a current leakage through the measuring instrument or a flow of highly mobile ions through the sample can produce spike signals in response to square wave mechanical loading. The effect of the impedance matching on the shape of the generated electromechanical potential has been demonstrated both analytically and experimentally $[7,8]$. To avoid the problem of artifacts arising form the shunt capacitance across the sample, the so-called null technique has been employed in which a charge opposite in sign to the generated piezoelectric charge is fed back to the sample until zero voltage appears across the sample electrodes [9]. Until recently, the measurements of inverse piezoeffect have been impossible due to the smallness of the corresponding piezoelectric constants ( $\sim 1-5 \mathrm{pm} / \mathrm{V}$ ), necessitating interferometric or scanning probe-based measurements. However, in both cases, fundamental studies of piezoelectricity in biological systems such as calcified and connective tissues have been limited by the complex hierarchical structures of biological materials, precluding quantitative electromechanical measurements.

In this chapter, we demonstrate how scanning probe microscopy (SPM) can be applied to nanoscale imaging and characterization of electromechanical behavior in biological systems. We employ piezoresponse force microscopy (PFM) to perform direct real-space imaging of biological samples at a hierarchy of length scales down to the nanoscale. A variety of biological systems, including human tooth, canine femural cartilage, deer antler, and butterfly wing, have been studied to demonstrate the general applicability of PFM for probing the bioelectromechanical behavior at the nanoscale. PFM is also compared to atomic force acoustic microscopy (AFAM) to illustrate the fact that the unique image formation mechanism in PFM results in significantly lower topographic cross-talk, thus allowing high-resolution imaging even of rough surfaces. One of the most striking examples includes visualization of the spiral shape of protein microfibrils with 5-nm spatial resolution and delineation of different protein strands.

\section{Piezoelectricity in Biological Systems: Overview}

In piezoelectric materials, application of mechanical stress yields electrical charge (direct piezoelectric effect). If an electrical field is applied to a piezoelectric sample, it produces mechanical strain (converse piezoelectric effect). From thermodynamic considerations, the piezoelectric coefficients linking charge and stress and strain 
and field are equal. Therefore, in the absence of other electromechanical interactions, information on the piezoelectric properties obtained from observation of either direct or inverse piezoeffect is equivalent.

For linear piezoelectric material, the relationship between the components of strain, $x_{i}$, stress, $X_{j}$, electric displacement, $D_{i}$, and electric field, $E_{k}$, are

$$
\begin{aligned}
& x_{i}=s_{i j} X_{j}+d_{i k} E_{k}, \\
& D_{i}=d_{i j} X_{j}+\varepsilon_{i k} E_{k},
\end{aligned}
$$

where materials properties are described by the elastic compliances, $s_{i j}$; piezoelectric constants, $d_{i k}$; and dielectric constant, $\varepsilon_{i k}$, tensors. The number of nonzero elements in matrix of piezoelectric coefficients, $d_{i j}$, strongly depends on symmetry of the crystalline material [10]. Notably, the piezoelectric constants form a thirdrank tensor, and thus exhibit much stronger orientation dependence then dielectric constants and elastic compliances, described by rank 2 and rank 4 tensors, respectively. For example, the rotation of the crystal by $180^{\circ}$ will not affect dielectric and mechanical behavior, but the signs of piezoelectric constants will reverse.

In biological materials, piezoelectric properties are related to the presence of oriented biopolymer molecules, such as polypeptides, polysaccharides, and polynucleotides, the structure of which are characterized by non-centrosymmetric point groups. The piezoelectric effect in biopolymers has been first discovered in wood by Shubnikov [11] and has been ascribed to the piezoelectric behavior of cellulose fibers. The piezoelectric nature of cellulose is assumed to be associated with the orientational displacement of hydroxyl groups. Detailed measurements show that the electric potential has been generated when applying a shear stress along the fiber axes [1]. It has been suggested that the observed behavior is due to point symmetry of $\mathrm{D}_{\infty}(\infty 2)$ of the fibers and that the piezoelectric tensor can be represented as

$$
\left[\begin{array}{cccccc}
0 & 0 & 0 & d_{14} & 0 & 0 \\
0 & 0 & 0 & 0 & -d_{14} & 0 \\
0 & 0 & 0 & 0 & 0 & 0
\end{array}\right]
$$

An appreciable piezoeffect has been observed in specially prepared wood sheets of oriented cellulose fibers: the obtained values of $d_{14}$ vary in the range from $3.1 \times$ $10^{-14} \mathrm{~m} / \mathrm{V}$ to $6.1 \times 10^{-14} \mathrm{~m} / \mathrm{V}$ depending on the type of wood. The values of the measured piezoelectric coefficients depend on many factors, such as the degree of orientation of the fibers, amount of the cellulose, and the density of fiber packing.

Systematic measurements of the piezoelectric properties of biopolymers have been undertaken by Fukada, who studied piezoelectricity in bones, tendon, silk, and other crystalline polymers $[4,12,13]$. The electromechanical behavior of bone attracted particular attention due to possible clinical application of electrical stimulation for bone growth and repair.

The two main components of bone and other calcified and connective tissues are the mineral hydroxyapatite (HAP) and collagen. The unit cell of HAP, $\mathrm{Ca}_{10}\left(\mathrm{PO}_{4}\right)_{6}(\mathrm{OH})_{2}$, belongs to the centrosymmetric space group $\mathrm{P}_{3} / \mathrm{m}$ and, 
therefore, cannot exhibit piezoelectric behavior. In collagen, polypeptide chains are arranged in a coiled-coil triple helix [14] forming a long rod-like molecule. Each individual molecule has a discernible short-range crystallinity. The collagen molecules presumably have $C_{6}$ [5] symmetry (sometimes $C_{3}$ symmetry is used [15]), which make them responsible for piezoelectricity in bone. The piezoelectric behavior of collagen can be described by the following matrix [5]:

$$
\left[\begin{array}{cccccc}
0 & 0 & 0 & d_{14} & d_{15} & 0 \\
0 & 0 & 0 & d_{15} & -d_{14} & 0 \\
d_{31} & d_{31} & d_{33} & 0 & 0 & 0
\end{array}\right] .
$$

This conclusion has been supported by the experimental evidence that demineralized bone does not show any piezoelectric activity [16]. Measurements of the converse piezoeffect in dry bones yielded nonzero value only for shear response with the effective values for $d_{14}$ and $d_{25}$ of the order of $10^{-13} \mathrm{~m} / \mathrm{V}$ and for $d_{15}$ and $d_{24}$ one order of magnitude less [3]. The $d_{31}$ and $d_{33}$ coefficients are even smaller (of the order of $10^{-14} \mathrm{~m} / \mathrm{V}$ ) [17].

The hexagonal symmetry $C_{6}$ has a polar axis, which means that the material described by Eq. (4) should also exhibit pyroelectric effect. The pyroelectric behavior has been observed in bone and tendon $[18,19]$ with the pyroelectric coefficient of bone to be $(0.4 \pm 0.2) 10^{-10} \mathrm{Coul} / \mathrm{m}^{2} \mathrm{~K}$. Some authors discussed a possible electret and ferroelectric behavior in bone [20,21].

Although several mechanisms responsible for the piezoelectric response in bone have been proposed, the most viable ones seem to be (1) distortion of the H-bonds in collagen or its cross-linkages and (2) stress-induced change in the spontaneous polarization of collagen $[5,7,18]$.

Experimental observations demonstrated some controversy regarding quantification of piezoelectricity in bones and showed the need for reasonable ranges of experimental parameters to perform realistic characterization of the piezoeffect in bones. Piezoelectric measurements done in Haversian systems (bones) show that that the piezoelectric tensor has hexagonal symmetry with the polar axis nearly parallel to the long axis of the bone [5]. On the other hand, in wet bone, the measurements showed a lack of symmetry [22]. Relative humidity and water content in bone were found to have a large influence on the electromechanical properties [23]. When water is absorbed by the system above the critical level, the dielectric constant showed a characteristic conductivity dispersion suggesting an increase in ionic mobility. In addition, a high level of polarization has been measured in wet bone that has been attributed to the streaming potential due to the fluid movement of ions in the microcapillary regions in bone.

The biological significance of piezoelectricity in bone is that it suggests correlation between bone properties and its functionality. Subsequently, the electromechanical behavior of bone attracted significant attention due to possible clinical application of electrical stimulation to bone growth and repair. It has been hypothesized that the piezoelectric behavior in bone provides a physical background to Wolff's law, which states that the architecture of bone depends on its function and that a change in function (i.e., change in the applied mechanical stress) will 
bring about a change in architecture. It is assumed that the piezoelectric matrix of bone transforms the mechanical stress into polarization charges. These charges precipitate the enzymatic activity, which results in bone growth or resorption in such a way as to accommodate the applied stress and minimize the piezoelectric response $[9,24,25]$. In spite of the plausibility of this hypothesis for explanation of Wolff's law, it is still not proven experimentally. Most general observations of piezoelectric behavior in bone can be summarized as follows: (a) stressed bone exhibits electronegativity in areas of compression, and (b) living nonstressed bone exhibits electronegativity in areas of bone growth and healing [26]. Despite the large number of publications in this area, the relationship between piezoelectricity and biological functionality has been elusive.

The most serious problem in studying biopiezoelectricity is that biological materials are composed of dissimilar structural elements arranged in a complex hierarchical structure, each level bringing new aspects to the overall properties of the material. In bone, arrangement of mineralized collagen fibrils gives rise to as many as seven levels of structural organization [27]. As a result, macroscopic piezoelectric properties of bone, that represent the response of a number of variously oriented collagen molecules, are dependent on the crystalline orientation of the sample and as well as on its position in the bone architecture $[28,29]$. Consequently, macroscopic piezoelectric coefficients measured in an arbitrary bone sample cannot be reliably compared with the piezoelectric measurements done in another sample. Several attempts have been undertaken to quantify the electromechanical behavior in bone by taking into account the specific form of the crystallite distribution and calculating the "standard equivalent single-crystal structure of bone" $[17,30]$. However, the exact piezoelectric tensor of "single-crystalline" collagen is not known.

Therefore, it is widely recognized that macroscopic studies of the electromechanical properties in biological systems are inherently limited by the hierarchical structure of these materials, thus necessitating the real space studies of electromechanical properties of biological systems from mesoscopic to molecular levels.

\section{PFM and AFAM for Studying the Ultrastructure of Biological Systems}

Details of the PFM experimental method can be found in this book as well as in earlier review papers [31-34]. Briefly, in PFM, application of a periodic electrical bias, $V_{t i p}=V_{d c}+V_{a c} \cos \omega t$, between the conductive SPM tip and the backside of the sample results in a periodic displacement of the surface, $d=d_{1 \omega} \cos (\omega t+\varphi)$, that can be measured with sub-Angstrom precision. The interaction volume beneath the tip (the volume that is piezoelectrically excited) depends on the contact radius, the applied bias, and local properties of the material, and is generally of the order of 5-20 nm, providing the measure of spatial resolution and field penetration in the material. The amplitude and phase of the cantilever oscillations reveal 
the information on the strength and sign of the local electromechanical response, respectively. Both vertical and lateral components of surface displacement can be measured [35] providing information on the normal and in-plane components of the electromechanical response vector. The image formation mechanism in PFM has been analyzed in detail [34] and it has been shown that in the absence of a dielectric gap between the tip and the surface, the PFM signal magnitude is independent of the contact area. The latter, however, determines the lateral spatial resolution. Recently, PFM has been applied to characterization of electromechanical properties of biological systems and to nanostructural imaging of a variety of protein-containing materials [36-38].

A complementary approach for structural imaging can be based on local mechanical scanning probe techniques, such as AFAM [39], which can distinguish dissimilar components of calcified tissues based on the difference in mechanical properties. In AFAM, the sample is vibrated mechanically by a piezoelectric actuator and acoustical waves transmitted to the tip are detected, providing a contrast between hard and soft regions of the sample. In both cases, in the small signal limit, the AFAM signal is related to the effective spring constant of the tip-surface junction, from which the elastic modulus of material can be determined. However, AFAM imaging of topographically inhomogeneous systems is limited by a significant topographic cross-talk due to the variations in local surface geometry (slope and local radius of curvature) that influences the contact mechanics of the tip-surface junction, thus precluding reliable elastic imaging on the nanoscale. Moreover, even for relatively flat surfaces, the AFAM signal is determined by the tip shape and radius of curvature, necessitating the use of calibration standards with known properties for quantitative measurements. Conversely, the PFM signal is virtually independent of the tip geometry provided that good contact between the tip and the surface is established, resulting in significantly less sensitivity of the technique for topographic cross-talk.

Below, we illustrate application of PFM and AFAM for high-resolution imaging of various calcified tissues, including dentin and enamel.

\subsection{Electromechanical Imaging of Human Teeth}

Teeth are composed of calcified tissues that have very different properties. The outer layer of tooth, enamel, is made up of hydroxyapatite (HAP) rods and a small fraction $(\sim 3-5 \%)$ of organic matrix component. It is hard and more brittle than the dentin tissue below that has significantly higher fraction (up to 30-40\%) of organic material, specifically type I collagen fibers interspersed with the HAP phase [40,41]. Dentin is formed by the growth tubules surrounded by randomly oriented HAP crystallites embedded in a collagen matrix. The interface zone between these two tissues, dentin-enamel junction (DEJ), plays an important role in preventing the crack propagation from enamel to dentin. Below, PFM characterization of dentin and enamel tissues in a dried human tooth has been performed in the vicinity of the DEJ. Before measurements, the crown cusp and the end of the root were cut using a low speed mechanical saw. Then, the cross sections of the sample were 

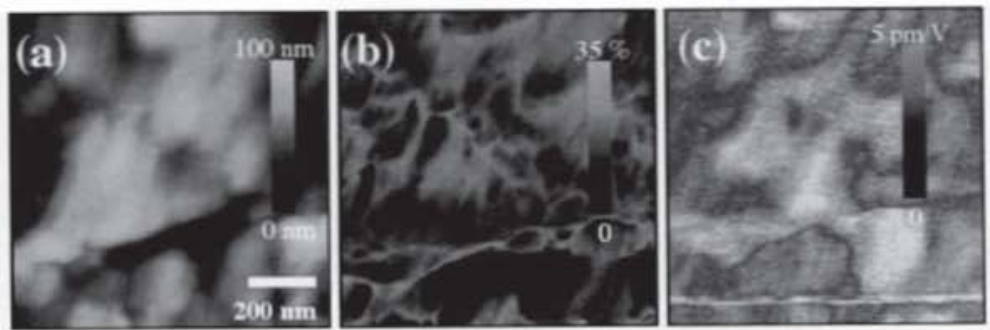

FIGURE 1. (a) Topographic, (b) AFAM, and (c) VPFM amplitude images of tooth dentin. Vertical scale in (b) and (c) is shown as a percentage of the average signal. Reprinted with permission from [42]. Copyright 2006, Elsevier, Inc.

polished to prepare a plane parallel plate of the thickness of about $500 \mu \mathrm{m}$. The sample has been fixed on a Si substrate using silver paint.

Prior to the PFM characterization, comparative PFM and AFAM imaging of tooth dentin has been performed. In Figure 1, it can be seen that the AFAM measurements provide much more detailed information on the internal tooth structure and allows visualization of features invisible on the topographic image. However, the image is strongly affected by the topographic cross-talk, where the grooves between the grains are associated with the bright features on images. Even though in some cases AFAM provides topography-independent contrast, in general topographic cross-talk due to changes in local surface curvature precludes unambiguous differentiation of tissue components. In comparison, vertical PFM image, which reveals significant variations of the contrast associated with dissimilar (organic or mineral) dental tissues, is not affected by the surface topography.

Figure 2 shows the results of PFM characterization of tooth dentin and enamel in the vicinity of the DEJ. While surface topography does not show any specific features related to these dissimilar tooth layers, PFM clearly illustrates a difference between dentin and enamel. Transition from the dentin layer with a high value of electromechanical signal (piezoelectrically active regions appear as bright spots in the amplitude PFM image) to the enamel layer with a very weak electromechanical
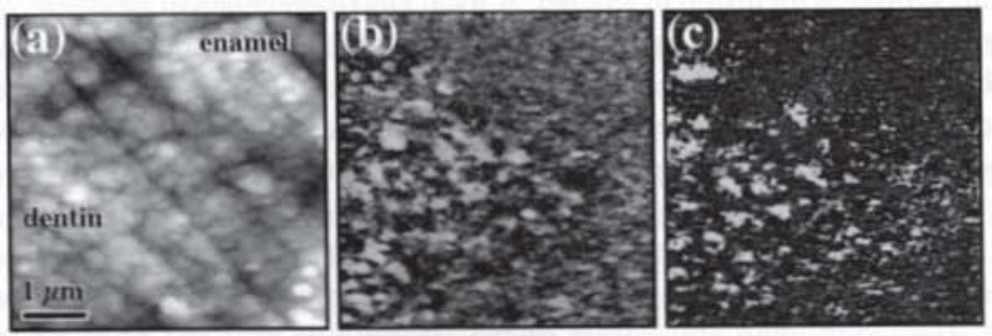

FIGURE 2. (a) Topographic, (b) VPFM phase, and (c) VPFM amplitude images of the tooth surface in the vicinity of the dentin-enamel junction (DEJ). 

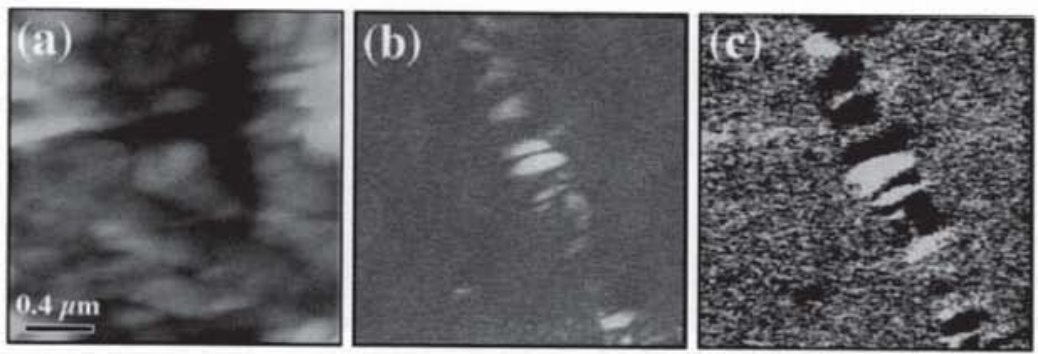

FIGURE 3. PFM imaging of a single protein microfibril in enamel in the vicinity of the DEJ: (a) topography, (b) VPFM amplitude, and (c) VPFM phase.

signal can be clearly seen in PFM. This behavior is consistent with the high fraction of piezoelectrically active protein components in dentin [43].

In the course of this study, we noticed that protein inclusions are quite common in the enamel in the vicinity of the DEJ. These inclusions have been also found at the larger distances from DEJ (up to $100 \mu \mathrm{m}$ ). These inclusions have complex internal structures that can be accessed by PFM. As an example, the PFM image of the enamel region close to the DEJ zone in Figure 3 shows a single protein chain cf about $100-150 \mathrm{~nm}$ in width embedded within a non-piezoelectric matrix. This result seems to be consistent with the earlier studies of the tooth morphology that found that organic fibers extend form the dentin zone through DEJ into the enamel [44]. It has been suggested recently [45] that the elastic properties of teeth to a large extent are determined by the structure of the intertubular dentin matrix although variations in the tubule density and orientation can also play a role [46].

\subsection{PFM Spectroscopy of Proteins}

Local electromechanical hysteresis loop measurement by PFM has been shown to be a powerful tool for characterization of switching phenomena in perovskite ferroelectrics $[32,33]$. A similar methodology has been applied for a point spectroscopy of a single protein inclusion in enamel. A local electromechanical response within a single domain region has been measured as a function of the modulation bias, $V_{a c}$. It has been observed that the tip oscillation amplitude is a linear function of modulation bias, as expected for a piezoelectric material [36]. From these measurements the effective piezoelectric coefficient was estimated to be about $0.15-0.25 \mathrm{pm} / \mathrm{V}$.

To check the ferroelectric behavior in these systems, the electromechanical response has been probed as a function of dc bias offset on the tip, $V_{d c}$. No inversion of the strain sign upon application of the opposite dc bias has been observed, indicating that, contrary to some early reports $[3,21,47]$, the protein fibers do not exhibit a ferroelectric behavior. 

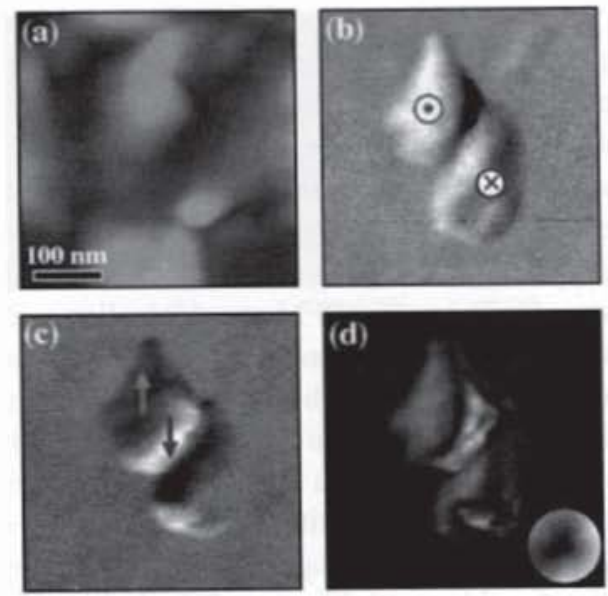

FIGURE 4. Orientational imaging of the protein fiber in tooth. (a) Surface topography of polished tooth enamel (vertical scale $20 \mathrm{~nm}$ ). (b) Vertical and (c) lateral PFM images of the same region as in (a) with a modulation bias of $10 \mathrm{~V}$ applied to the tip. A protein fibril embedded within a non-piezoelectric matrix can be clearly seen in the center of the PFM images. Circles and arrows in (b) and (c), respectively, show the orientation of the piezoresponse vector. (d) Vector PFM map of local electromechanical response. Color indicates the orientation of the electromechanical response vector, while the intensity provides the magnitude (color wheel diagram). (See also Plate 4 in the Color Plate Section.)

\subsection{High-Resolution PFM Imaging of Proteins in Dental Tissues}

To get further insight into the structure of the protein fibril, below we demonstrate the high-magnification imaging of enamel using two different modes of PFM, namely, lateral (LPFM) and vertical (VPFM) modes. Application of these modes allows acquisition of two components of the local piezoresponse vector-normal (vertical) and in-plane (lateral) components-and may potentially lead to the assessment of the crystallographic orientation of the protein molecules using the vector PFM approach developed previously for orientational imaging of polycrystalline piezoelectric materials [48].

Topographic image of the enamel surface in Figure 4(a) does not show any morphological features related to the protein presence. In comparison, both vertical and lateral PFM images show a strong electromechanical response (signal to noise ratio $>4: 1$ ) in the center that we attribute to a protein fibril embedded within a non-piezoelectric matrix (Figure 4(b,c)). The spatial resolution of PFM, determined as the half-width of the boundary between piezoelectric regions with different orientation, is about $5 \mathrm{~nm}$. Note that the resolution achieved is an order of magnitude better than 50-100 nm typical for single crystals and is comparable to the best results achieved to date for thin films of ferroelectric perovskites. In PFM in the strong indentation regime [34], the resolution is limited by the tip-surface 
contact area. For a tip-surface indentation force on the order of $\sim 100 \mathrm{nN}$, the contact radius can be estimated as $\sim 5-20 \mathrm{~nm}$ depending on the tip radius of curvature and effective Young's modulus of the material. Here, this limit is achieved for a biological material. Given the strong orientation dependence of the PFM signal, this opens a pathway to molecular orientation imaging at comparable resolution. Comparison of the VPFM and LPFM images shows a different pattern of piezoelectric domains, suggesting a complicated fibril structure, most likely consisting of several protein strands. The vector PFM approach allows combined representation of the VPFM and LPFM data within one image [37]. In this approach, VPFM and LPFM images are normalized with respect to the maximum and minimum values of the signal amplitude so that the intensity changes between -1 and 1 . Using commercial software (Mathematica 5.0, Wolfram Research) these 2D vector data $(v p r, l p r)$ are converted to the amplitude/angle pair, $A_{2 D}=\operatorname{Abs}(v p r+I l p r)$, $\theta_{2 D}=\operatorname{Arg}(v p r+I l p r)$. These sets of data can be plotted in one image using color representation so that the color will correspond to the orientation of the piezoresponse vector in the plane perpendicular to the cantilever axis (Figure 4(d), color inset) and thus will serve as a measure of the local protein fibril orientation. Color intensity in this case will correspond to the magnitude of the piezoresponse signal according to the color wheel diagram. The color encoded vector PFM map, shown in Figure 4(d), clearly delineates a helical structure, visualizing the electromechanically active protein fibril conformation in real space [49]. Note the additional details (complex spiral shape of the molecule) that can be visualized in the $2 \mathrm{D}$ vector PFM map, as compared to the original data sets.

Vertical and lateral PFM signals provide complementary information on the orientation of protein fibrils in dentin, thus allowing statistical description of the microstructure. Shown in Figure 5(a,b) are the VPFM and LPFM images of the $3 \times 3 \mu \mathrm{m}^{2}$ area of dentin, respectively, which exhibit strong PFM contrast due to the presence of protein rich regions. Figure 5(c) shows the double histogram of normalized VPFM and LPFM signals in the same dentin area, representing the count number of points with the signal level in the interval $(v p r+\delta v, l p r+\delta l)$, where $v p r, l p r \in(-1,1)$. Shown in Figure 5(d,e) are the amplitude and angle signal distributions. These data suggest that there are two primary antiparallel orientations of the piezoresponse vector. Thus, the local dentin microstructure can be well represented by axially ordered antiparallel protein fibers, as shown in inset on Figure 5(f). The characteristic fiber size can be determined using self-correlation function analysis, as is illustrated in Figure 5(f). The normalized experimental function can be well approximated using a simple phenomenological form $C(x)=$ $A \exp (-x / \xi)$, where characteristic domain size $\xi$ is $160 \pm 2 \mathrm{~nm}$. This analysis illustrates the reconstruction of local microstructure of peritubular dentin from the PFM data.

\subsection{Molecular Orientation in PFM}

The analysis described above can be further extended to create a semiquantitative nanoscale map of local molecular orientation. The electromechanical properties 


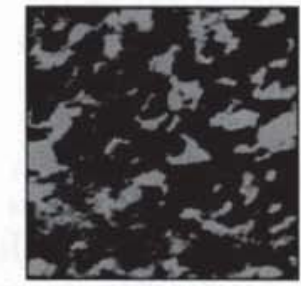

(a)

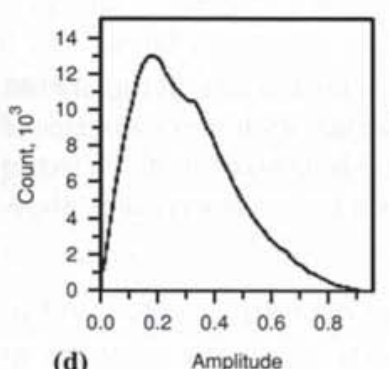

(b)

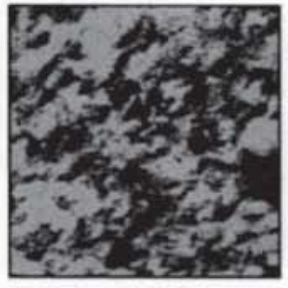

Leteral PFM

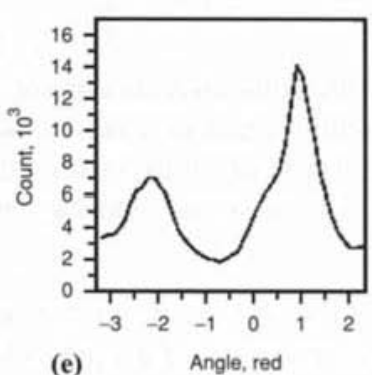

(e)

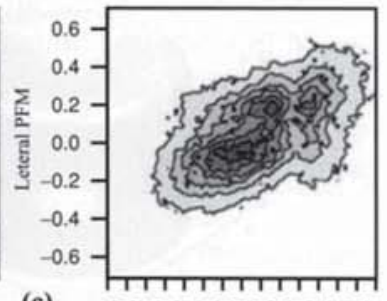

(c)

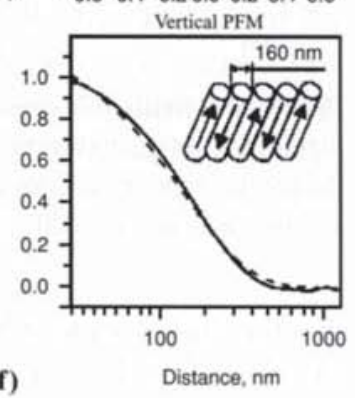

FIGURE 5. (a) VPFM and (b) LPFM phase images of the $3 \times 3 \mu \mathrm{m}^{2}$ region of dentin. (c) Double histogram of the vertical and lateral PFM signals of the same area of dentin shown in (a) and (b). (d) Amplitude and (e) phase distribution of the piezoresponse vector. (d) Selfcorrelation function for the PFM signal. Dotted line is a fitting using $C(x)=A \exp (-x / \xi)$, where characteristic domain size $\xi$ is $160 \mathrm{~nm}$. The inset shows the simplified nanostructural model for dentin formed by antiparallel protein fibrils. Reprinted with permission from [36]. Copyright 2005, American Institute of Physics.

of solids are characterized by a piezoelectric tensor, $d_{i j}$, where tensor elements are determined in the coordinate system linked to the principal crystallographic axes. On the other hand, the experimentally measured VPFM and LPFM signals are determined by the coefficients $d_{33}^{l}$ and $d_{34}^{l}$ of the piezoelectric tensor, $d_{i j}^{l}$, in the laboratory coordinate system [48]. The two coordinate systems are related by a set of three Euler rotation angles, $(\theta, \psi, \varphi)[50]$ that uniquely defines the local crystallographic orientation in the laboratory coordinate system. The relationship between the $d_{i j}^{l}$ tensor in the laboratory coordinate system and the $d_{i j}$ tensor in the crystal coordinate system is [50]:

$$
d_{i j}^{l}=A_{i k} d_{k l} N_{l j},
$$

where $N_{i j}$ and $A_{i j}$ are the rotation matrices. For materials with a known $d_{i j}$ tensor, the local crystallographic orientation $\left(\phi_{i}, \theta_{i}, \psi_{i}\right)$ can be derived by solving Eq. (5). The detailed theoretical analysis of electromechanical orientational imaging by vector PFM including approaches to calibration and measurement artifacts is developed elsewhere [48].

For the protein fibril studied in this work, only partial information on the electromechanical response vector is available from VPFM and LPFM data. Assuming 


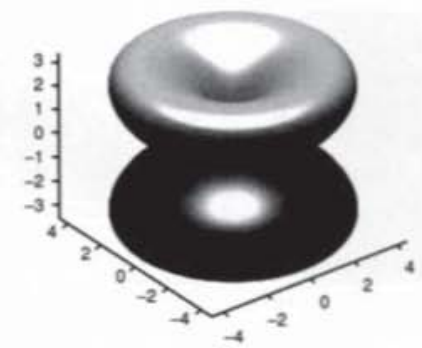

(a)

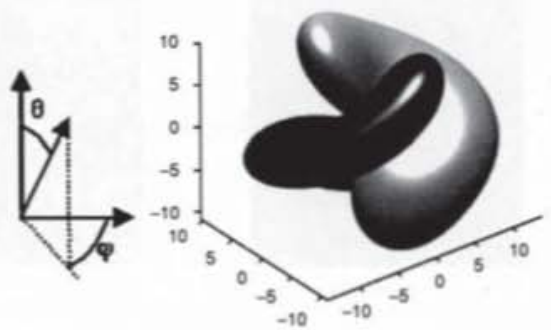

(b)

FIGURE 6. Orientation dependence of the absolute value of (a) vertical and (b) lateral PFM signals for collagen (bright $=$ positive; dark = negative). Note that while the exact shape of the piezoresponse surfaces will depend on precise values of the elements of the piezoelectric tensor, the nodes are determined by the crystal symmetry only. Image courtesy of S. Jesse.

that the fibril is formed by collagen, ${ }^{1}$ the $d_{i j}$ matrix has the form as shown in Eq. (4). Thus, from Eq. (5), the components of the piezoelectric tensor proportional to the PFM signal can be written as

$$
\begin{aligned}
& d_{33}^{l}=0.5 \cos \theta\left(d_{15}+d_{31}+d_{33}-\left(d_{15}+d_{31}-d_{33}\right) \cos 2 \theta\right), \\
& d_{34}^{l}=-\left(\left(d_{31}-d_{33}+\left(d_{15}+d_{31}-d_{33}\right) \cos 2 \theta\right) \cos \psi+d_{14} \cos \theta \sin \psi\right) \sin \theta, \\
& d_{34}^{l}=-\left(\left(d_{31}-d_{33}+\left(d_{15}+d_{31}-d_{33}\right) \cos 2 \theta\right) \sin \psi-d_{14} \cos \theta \sin \psi\right) \sin \theta .
\end{aligned}
$$

In this case, the molecule is rotationally invariant and the response is independent on the third Euler angle, $\varphi$. Orientation dependence of PFM signal surfaces are shown in Figure 6. Note that while the exact shape of response surfaces are strongly dependent on the $d_{i j}$, values the nodes are dependent only on the crystal symmetry and should be universal for most biological systems, which generally exhibit shear piezoelectricity.

For rotationally invariant molecules, the combination of VPFM and LPFM data is sufficient for reconstruction of molecular orientation. To calculate the effective electromechanical response measured in our PFM experiment, we use the $d_{i j}$ values (in $\mathrm{pm} / \mathrm{V}$ ) for tendon reported by Gunjian [17]:

$$
\left[\begin{array}{cccccc}
0 & 0 & 0 & -2.66 & 1.40 & 0 \\
0 & 0 & 0 & 1.40 & 2.66 & 0 \\
0.067 & 0.067 & 0.087 & 0 & 0 & 0
\end{array}\right] .
$$

Neglecting the $d_{33}$ and $d_{13}$ coefficients, we obtain that the VPFM and LPFM signals are proportional to $d_{33}^{l}=0.7 \cos \theta(1-\cos 2 \theta)$ and $d_{34}^{l}=(2.66 \cos \theta \sin \psi-$ $1.4 \cos 2 \theta \cos \psi) \sin \theta$, respectively. By solving these two linearly independent

\footnotetext{
${ }^{1}$ Note that collagen is chosen only as an example since there is no direct proof that the observed protein is indeed collagen.
} 
equations, we can determine angles $\theta$ and $\psi$. Given the large degree of uncertainty in published values for $d_{i j}$ and lack of absolute calibration, the VPFM and LPFM signals were normalized by taking $d_{33}^{l \max }=\max \left[d_{33}^{l}(\theta, \psi)\right]$ and $d_{34}^{l \max }=$ $\max \left[d_{34}^{l}(\theta, \psi)\right]$, where $(\theta, \psi) \cup(0,2 \pi)$. This provides a semi-quantitative calibration of the measured PFM signal that can be related to the local electromechanical properties of the collagen.

It should be noted that due to the absence of reliable piezoelectric data and incomplete information on the electromechanical response vector (only 2 of 3 orthogonal components have been measured) only semiquantitative analysis of the orientational imaging could be performed in this case. However, we expect that the potential for orientation imaging of biological systems at the nanoscale level will stimulate further experimental development of this approach (e.g., using complete 3D PFM data), and theoretical modeling of the electromechanical coupling coefficients for complex biomolecules, which can potentially open a pathway to molecular identification.

\section{Electromechanical Properties of the Butterfly Wings}

Butterfly wings exhibit a complex microstructure that is designed to generate certain color and pattern effects, provide flexural stiffness and deformability of the wing, control heat transformation, etc. [51-55]. The wings are covered by thousands of chitin scales that vary in shape, size and color. Scale microstructure has been the subject of numerous studies by optical and electron microscopy techniques $[56,57]$. Examination of the wing structure yields information on the interference and diffraction mechanisms that produce certain color patterns of the wings. Furthermore, the intricate wing structure also provides an inspiration for engineering of complex light-weight deformable structures that can be used in micromechanical devices. In this section, we demonstrate how the SPM-based approach for characterization of butterfly wings can go beyond visualization of the scale structure and can actually allow evaluation of their elastic and electromechanical properties on the length scales from $50 \mu \mathrm{m}$ to $10 \mathrm{~nm}$. Examination of the piezoelectric properties of the butterfly wings at the nanoscale can be used as a way for elucidation of their ultrastructure by employing the electromechanical activity of the chitin fibers.

A sample used in this study has been cut from the dorsal forewing of Vanessa virginiensis butterfly (Figure 7(a)). The sample has been mounted on the $\mathrm{SiO}_{2} / \mathrm{Si}$ wafer using silver paint. Optical micrograph in Figure 7(b) shows a number of white-pigmented scales covering the area of the wing that was subsequently inspected by SPM. Mesh-like structure of longitudinal lamellae connected by crossribs can be seen in the topographic images (Figure 7(c)). It is believed that this structure is a basis for high structural stability of the scale.

Figure 7(d) shows another image of the same area of the wing as in Figure 7(c) acquired by AFAM. The AFAM signal is directly related to the mechanical properties of the sample below the tip, providing an approach to visualize, if not quantify, 

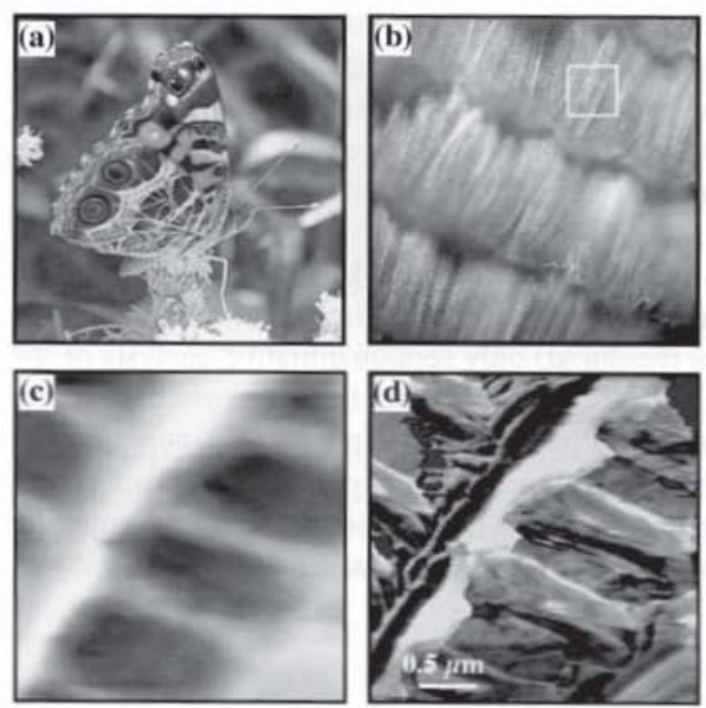

FIGURE 7. (a) Optical photograph of Vanessa virginiensis (courtesy of Jeffrey Pippen, Duke University) and (b) optical micrograph of the wing scales. (c) AFM surface topography of the wing (vertical scale is $1 \mu \mathrm{m}$ ). (d) AFAM elasticity image (vertical scale is $18 \%$ of the average signal) of the wing obtained simultaneously with (c). (See also Plate 5 in the Color Plate Section.)

the variations in mechanical properties at the nanometer scale. Figure $6(d)$ shows significant variations in the elastic constant within an individual ridge that appear as regions of different contrast, suggesting an internal structural inhomogeneity. Regions of higher contrast in AFAM correspond to harder material. Note the difference between effective resolution on the topographic and AFAM images-while no features smaller than $100 \mathrm{~nm}$ can be distinguished in the topographic image, the AFAM image shows details with sub-10-nm resolution. This result illustrates the possibility of addressing the structural differences in the protein-chitin matrix constituting the scale by means of various modes of SPM.

Butterfly wings, like exoskeletons of most insects, represent a form of biological composite made of chitin fibers in a protein matrix. Similar to many other polysaccharide-based biopolymers, chitin is reported to be piezoelectric [58-60]. However, the complex nanoscale structure and lack of macroscopic samples have previously hindered studies of piezoelectricity in such systems. Here, the electromechanical properties of the butterfly wing have been probed by PFM by scanning a $2 \times 2-\mu \mathrm{m}^{2}$ wing region. Remarkably, vertical PFM image in Figure 8(a) clearly shows piezoelectric contrast that we attribute to the electromechanical behavior of chitin.

Vertical PFM (VPFM) measurements have been complemented by the lateral LPFM imaging, providing the information on two components of electromechanical response vector. Shown in Figure $8(b)$ is the LPFM image obtained from the same area of the wing as the VPFM in Figure 8(a). In Figure 8(c), the 2D-vector PFM image illustrates the position-dependent piezoelectric properties: the magnitude and orientation of the electromechanical response vector of the wing scale in the plane perpendicular to the cantilever long axis (color inset). Here, red color corresponds to the preferential orientation of chitin fibers in the direction normal to the surface while green color indicates the primarily in-plane orientation of 

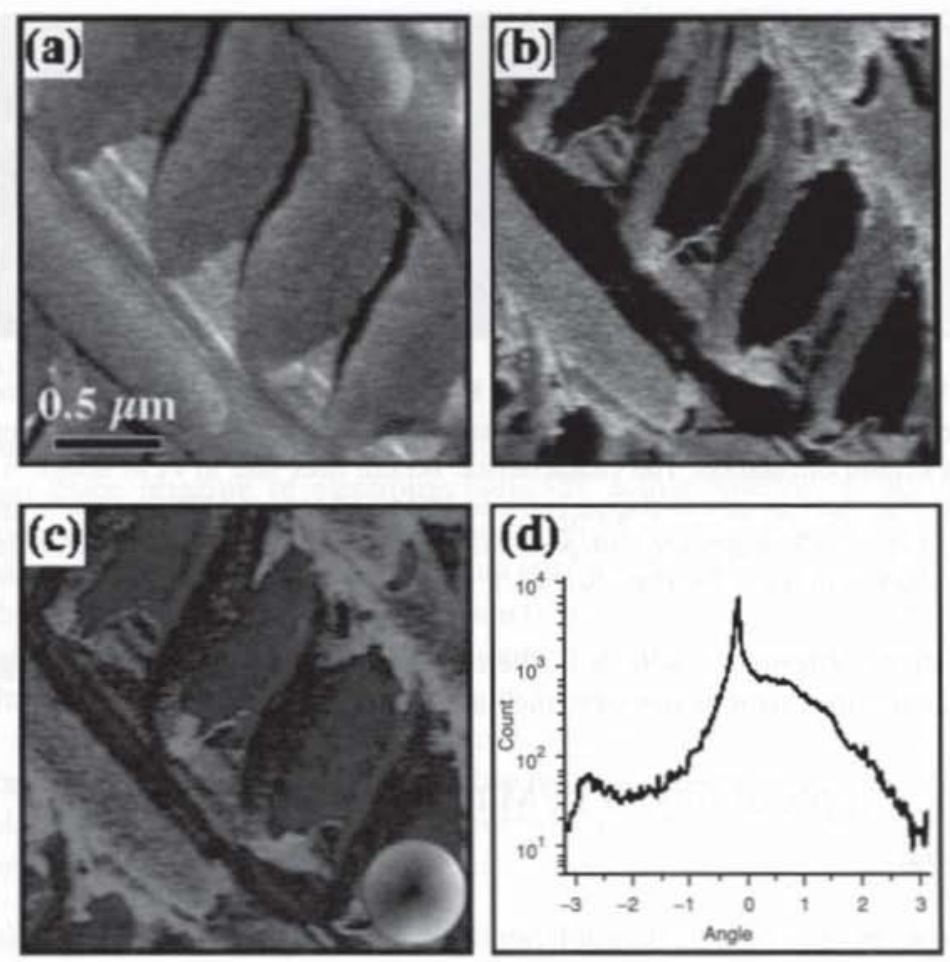

FlGURE 8. (a) Vertical and (b) lateral PFM images of the same area of the wing. (c) Color representation of the VPFM and LPFM data. Note the presence of the strong positiondependent PFM signal, indicative of the piezoelectric properties of the butterfly wing. (d) Angle distribution histogram of data in (c) that indicates the predominant orientation of the chitin fibers in the wing scale. (See also Plate 6 in the Color Plate Section.)

the fibers. If we assume that the piezoelectric tensor of chitin exhibits shear piezoelectric response (as in collagen), then the non-zero VPFM signal observed in the butterfly wing should lead to a conclusion that the chitin molecules are oriented at some angle to the surface. Note also that color is virtually uniform within the ridge and cross-rib, while varying between these structural elements indicating an orientational difference of about $90^{\circ}$. The histogram analysis of angle distribution of the 2D PFM signal in Figure 8(c) reveals two peaks corresponding to two main orientations of chitin fibers within lamellae and cross-ribs, respectively (Figure 8(d)).

No quantitative data has been obtained yet, however, we estimate the effective piezoelectric constant to be just below $1 \mathrm{pm} / \mathrm{V}$. How essential are the piezoelectric properties for functionality of the butterfly wings is an open question that needs to be addressed in future research. In the meantime, the piezoelectric properties of the wing can be utilized to examine its local structure at the micro- and nanoscale. The obtained results demonstrate that the SPM-based methods have a great potential in improving the understanding the structure-property-functionality relationship 

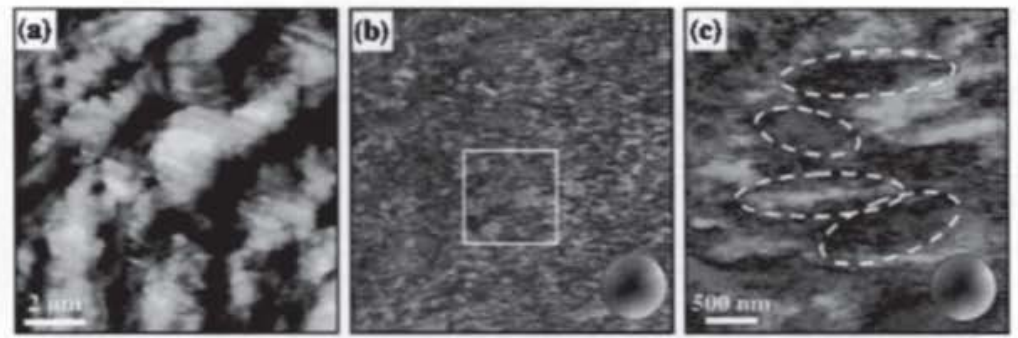

FIGURE 9. Surface topography (a) and vector PFM images (b,c) of deer antler. Vector PFM illustrates finer details of internal antler structure, including the presence of region with different keratin orientation. The characteristic keratin fiber size in PFM image is $\sim 200$ $\mathrm{nm}$. Note that there is no correlation between PFM and topographic images, suggesting absence of cross-talk. Reprinted with permission from [42]. Copyright 2006, Elsevier, Inc. (See also Plate 7 in the Color Plate Section.)

in biological systems, as well as in the development and testing of biologically inspired micromechanical devices, such as biomimetic wings.

\section{Wider Applicability of PFM to Bioelectromechanical Imaging}

Data shown below illustrate the applicability of PFM for structural characterization to a broader range of materials system. Figure 9(a) illustrates surface topography of a longitudinal cross-section of the deer antler. Unlike teeth, this material contains lipids, which can diffuse to the surface thus forming a non-conductive layer and significantly complicating PFM imaging. This necessitates the use of cantilevers with relatively high spring constants $(>5 \mathrm{~N} / \mathrm{m})$ to penetrate the contamination layer. To visualize electromechanical response data, we again employ color representation of the PFM signal acquired from the same area (Figure 9(b), color inset), which allows visualization of otherwise invisible microstructural elements. Vector PFM image of a smaller area (Figure 9(c)) clearly shows elongated regions of homogeneous contrast (marked by white ellipses) about 2-3 $\mu \mathrm{m}$ long and 200-300 $\mathrm{nm}$ wide which are presumably due to the keratin fibrils of different orientation.
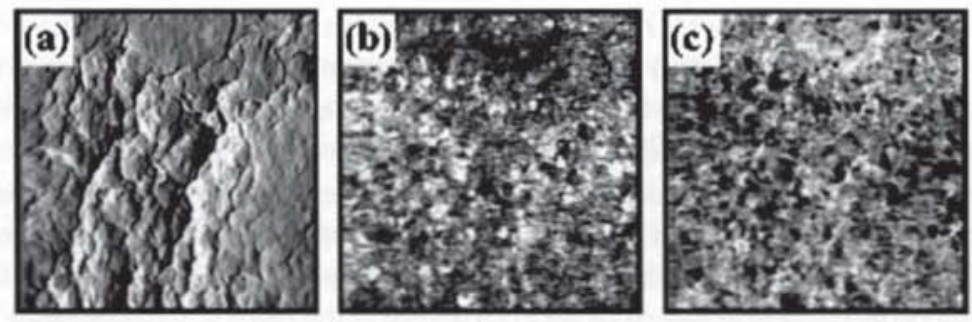

FIGURE 10. (a) Surface topography and vertical PFM (b) amplitude and (c) phase images of the microtomed canine femoral cartilage. The scan size is $2 \times 2 \mu \mathrm{m}^{2}$. 
Finally, shown in Figure 10 is a set of topographic and PFM images of canine femur cartilage. The surface is formed by multiple mounds with characteristic size of 100-200 nm, formed during the drying and shrinking of the cartilage surface. Vertical PFM phase and amplitude images clearly indicate that the surface is piezoelectric. Bright regions in the PFM amplitude image are associated with the piezoelectrically active collagen embedded in the extracellular matrix.

\section{Conclusion}

To summarize, piezoresponse force microscopy is shown to be a powerful tool for real-space imaging of electromechanically active proteins in calcified and connective tissues with nanoscale resolution. PFM allows differentiation between organic and mineral components and is not sensitive to topographic cross-talk, thus significantly simplifying the interpretation of image contrast in terms of materials microstructure. It has been shown that application of PFM provides an additional insight into the composition and structure of dental tissues. It is suggested that PFM can be used to study internal structure and orientation of the protein microfibrils in the calcified matrix. PFM thus holds a great promise for imaging and elucidating the structure/property relationship in biological tissues as well as in the development and testing of biologically inspired materials and devices.

\section{References}

1. V. A. Bazhenov, Piezoelectric Properties Of Wood (Consultants Bureau, New York, 1961).

2. E. Fukada and I. Yasuda, J. Phys. Soc. Jpn. 12, 1158 (1957).

3. M. A. El Messiery, IEE Proc. 128 A, 336 (1980).

4. E. Fukada, J. Phys. Jpn. 10, 149 (1955).

5. E. Fukada, Biorheology 5, 199 (1968).

6. E. Fukada, Biorheology 32, 593 (1995).

7. M. H. Shamos, and L. S. Lavine, J. Clin. Orthop. 35,177 (1966).

8. W. Williams, and L. Breger, J. Biomech. 8, 407 (1975).

9. A. J. Bur, J. Biomech. 9, 495 (1977).

10. W. G. Cady, Piezoelectricity: An Introduction to the Theory and Applications of Electromechanical Phenomena in Crystals (New York: Dover Publications, 1964).

11. A. V. Shubnikov, Piezoelectric textures, USSR Academy of Sciences, Moscow, 1946.

12. E. Fukada, Rep. Progress in Polymer Phys. Jpn. 3, 163 (1960).

13. E. Fukada, J. Phys. Soc. Jpn. 12, 1301 (1956).

14. M. Yamauchi, Advances in Tissue Banking 6, 445 (2002).

15. G. N. Ramachandran and G. Kartha, Nature 174, 269 (1954).

16. A. A. Marino, and R. O. Becker, Calc. Tissue Res. 8, 177 (1971).

17. A. A. Gundjian, and H. L. Chen, IEEE Transactions on Biomedical Engineering 21 (3), 177 (1974).

18. S. B. Lang, Nature 212, 704 (1966).

19. A. R. Liboff, and M. Frust, Ann. NY Acad. Sci. 238, 26 (1974). 
20. S. Mascarenhas, in Electric Charge Storage, ed. by M. Perlman (The Electroemechanical Society Inc., 1972), pp. 650-656.

21. H. Athenstadt, Naturwiss 48, 465 (1961).

22. J. C.Anderson, and C. Eriksson, Nature 227, 491 (1970).

23. T. G. Netto, and R. L. Zimmerman, Biophysical J. 15, 573 (1975)

24. C. A. L. Bassett, Calc. Tiss. Res. 1, 252 (1968).

25. A. Gjelsvik, J. Biomech. 6, 69 (1973).

26. C. T. Brighton, Z. B. Friedenberg, E. I. Mitchell, and R. E. Booth, Clin. Orthop. Relat. Res. 124, 106 (1977).

27. S. Weiner and H. D. Wagner, Annu. Rev. Mater. Res. 28, 271 (1998).

28. H. Athenstadt, Nature 238, 830 (1970).

29. W. S. Williams, M. Johnson, and D. Gross, in Electrical Properties of Bone and Cartilage, ed. by Brighton, Black and Pollack (Grune and Stratton, 1979).

30. E. Korostoff, J. Biomech. 10, 41 (1977).

31. A. Gruverman, in Encyclopedia of Nanoscience and Nanotechnology, ed. by H. S. Nalwa, Vol. 3, pp. 359-375 (American Scientific Publishers, Los Angeles, 2004).

32. A. Gruverman, O. Auciello, and H. Tokumoto, Annu. Rev. Mat. Sci. 28, 101 (1998).

33. Nanoscale Characterization of Ferroelectric Materials, ed. by $M$. Alexe and A. Gruverman (Springer-Verlag, Berlin 2004).

34. S. V. Kalinin, E. Karapetian, and M. Kachanov, Phys. Rev. B 70, 184101 (2004).

35. L. M. Eng, H.-J. Guntherodt, G. A. Schneider, U. Kopke and J. M. Saldana, Appl. Phys. Lett. 74, 233 (1999).

36. S. V. Kalinin, B. J. Rodriguez, S. Jesse, T. Thundat, and A. Gruverman, Appl. Phys. Lett. 87, 053901 (2005).

37. S. V. Kalinin, B. J. Rodriguez, J. Shin, S. Jesse, V. Grichko, T. Thundat, A. P. Baddorf, and A. Gruverman, Ultramicroscopy 106, 334 (2006).

38. C. Halperin, S. Mutchnik, A. Agronin, M. Molotskii, P. Urenski, M. Salai, and G. Rosenman, Nano Letters 4, 1253 (2004).

39. U. Rabe, M. Kopycinska, S. Hiserkorn, J. Munoz-Saldana, G.A. Schneider, and W. Arnold, J. Phys. D 35, 2621 (2002).

40. M. Goldberg, M. Takagi, Histochem. J. 25, 781 (1993).

41. A. Linde, S. P. Robins, Coll. Relat. Res. 8, 443 (1988).

42. B. J. Rodriguez, S. V. Kalinin, J. Shin, S. Jesse, V. Grichko, T. Thundat, A. P. Baddorf, and A. Gruverman, J. Struct. Biol. 153, 151 (2006).

43. A. A. Marino, B. D. Gros, Archs. Oral Biol. 34, 507 (1989).

44. S. Habelitz, M. Balooch, S. J. Marshall, G Balooch, G. W. Marshall, J. Struct. Biol. 138, 227 (2002).

45. J. H. Kinney, M. Balooch, G. W. Marshall, S. J. Marshall, Arch. Oral Biol. 44, 813 (1999).

46. H. Sano, B. Ciucchi, W. G. Matthews, D. H. Pashley, J. Dent. Res. 73, 1205 (1994).

47. M. A. Elmessiery, G.W. Hastings, and S. Rakowsky, J. Biomed. Eng. 1, 63 (1979).

48. S. V. Kalinin, B. J. Rodriguez, S. Jesse, J. Shin, A. P. Baddorf, P. Gupta, H. Jain, D. B. Williams, and A. Gruverman, Microscopy and Microanalysis 12, 206 (2006).

49. J. W. Smith, Nature 219, 157 (1968).

50. R. E. Newnham, Properties of Materials: Anisotropy, Symmetry, Structure, Oxford University Press, 2005.

51. R. J. Wootton, Scient. Am. 262, 114 (1990).

52. C. P. Ellington, J. Exp. Biology 202, 3439 (1999).

53. R. J. Wootton, Proc. R. Soc. Lond. B 262, 181 (1995). 
54. R. Dudley, J. Exp. Biol. 150, 37 (1990).

55. S. J. Steppan, J. Res. Lepidoptera 35, 61 (2000).

56. H. Tada, S. E. Mann, L. N. Miaoulis, and P. Y. Wong, Applied Optics 37, 579 (1998).

57. P. Y. Wong, T. H. Gupta, M. C. B. Robins, and T. L. Levendusky, Optics Letters 28, 19 (2003).

58. C. C. Silva, C. G. A. Lima, A. G. Pinheiro, J. C. Goes, S. D. Figueiro, and A. S. B. Sombra, Phys. Chem. Chem. Phys. 3, 4154 (2001).

59. H. Maeda, Biophysical J. 56, 861 (1989).

60. E. Fukada, R. L. Zimmerman, and S. Mascarenhas, Biochem. Biophys. Res. Comm. 62, $415(1975)$. 